\title{
Research on the Application of Organizational Management Information System in Large Scale Sport Event
}

\author{
Zhi-mei Zhang \\ Institute of P.E.,Zhongzhou University,Zhengzhou 450044,China \\ zhangzhimei0508@sina.com
}

Keywords: Organizational management information system, Project management, Large scale sport event

\begin{abstract}
To well organize China's Large-scale Sport Events, causes the Large-scale Sport Events organization to manage in the process each kind of activity and the work carries on the effective classification the equipment personnel and the division of labor authorization, we synthesized research techniques and so on utilization systems science, management science, mathematics modeling, artificial intelligence, information technology design in China's Large-scale Sport Events to organize the management information system, This article comprehensively elaborated this system function demand, the bare bone, the system design and the core function and so on.
\end{abstract}

\section{Introduction}

Sport events have a great influence on the society, policy, economy, travel, nature and environment, so many countries and districts are trying to get more profit by holding kinds of sport events. The important value of sport events lies in bringing advantage to the countries or the districts. The sport events of our country were held by the government and had many questions such as the lacking of funds, ignoring the market, but now we realize it. So it is necessary for our country to find out an efficient management method [1]. Under the international sports event globalization development background, with the unceasing development, economy and society of our country, in our country's whole sports developmental strategy, especially in gravity change of the sports developmental strategy center in recent years, sports industry, becomes the new sports developmental strategy the hot spot which takes the sports event as the backing, looks the society and the economic development as the main purpose, and drawing expends and develops the new economic growth as the essential target [2]. That large-scale sports event which has the public welfare attribute, becomes the best choice for all levels of the government to promote the urban development and to improve the urban image. This has directly caused the vigorous growth of sports event practices in our country. However, compared with vigorous development of sports event practices and the management level existed obvious lag, especially the large-scale sports event's management and organization is the weakness and the difficulty.

A sport event is a special event that has the characters of complicity, competition, multi-goals and objects and merchandise with strong organization culture and potential marketing background. A sport event can be classified into a project [3]. It also supplies competition goods and relative service goods to meet various stakeholders' needs. Any sport event can be formed by three-dimensional description. Sport events have strong impact on society and culture, nature and environment, politic, tourism and economy on some circumstances [4].

The process of sport event operation is to manage the input to output. Sport event operation must include the considerations of marketing that embraces goods marketing and service, humanization and environment, contingency and organizational behaviors. Operation tasks include budget and control, risk management, human resources management, logistics, communication, marketing and competition. Manageable factors are divided into two kinds, which include supporting factors and promoting factors.

The process of sport event operation is consisted of planning, organizing, implementing and evaluating [5]. The model has two sub-models: one is a vertical model, which happens during the 
preparing time, the other is a horizontal model, which happens during staging time. Sport event's goal and objects decide the importance of each task. Integration marketing includes the sponsorship, advertisement, media broadcasting, courteous reception, public relationship and sales. Sport event operation model includes project start, planning and organizing, implementing and evaluating. Our country's sport event is affected by government's structure and mechanism, especially the competition institution, and manager's stuff. Although marketing operation is received more attention, marketing methods must be researched fully. The Chinese operation model in sport events includes project start, planning and organizing, and implementing and evaluating.

\section{The basic framework of Management Information System}

Integrated use of systems science, management science, mathematical modeling, artificial intelligence, IT and other research methods in this study, a detailed analysis of the Large-scale Sport Events held in various work flow, information flow, a mathematical model based on detailed important issue of the functional requirements of the discourse of Large-scale Sport Events organization and management information systems, the basic framework of the overall design and core functionality, and implementation steps.

Large-scale Sport Events organization and management information systems departments of Large-scale Sport Events management information system, we must rely on existing electronic information systems in various departments of the Large-scale Sport Events, architecture. Organization and management of Large-scale Sport Events can be divided into strategic level, management level, knowledge level and business level, four levels, Shown in Fig. 1. In the Large-scale Sport Events organization and management system, various types of senior management responsible for the leadership and decision-making functions; all kinds of middle management agencies and knowledge and data staff coordinating role of the organization's work; Command and coordination of all types of organizations are responsible for their professional work of the Organization. Various organizations at all levels of organization and management of organic linking information, Therefore, the Large-scale Sport Events organization and management system is the basic guarantee of fast, efficient work of the organization.

In order to achieve information sharing among all levels of various types of Large-scale Sport Events organization and management department, Large-scale Sport Events organization and management information systems need to develop information-sharing standards.

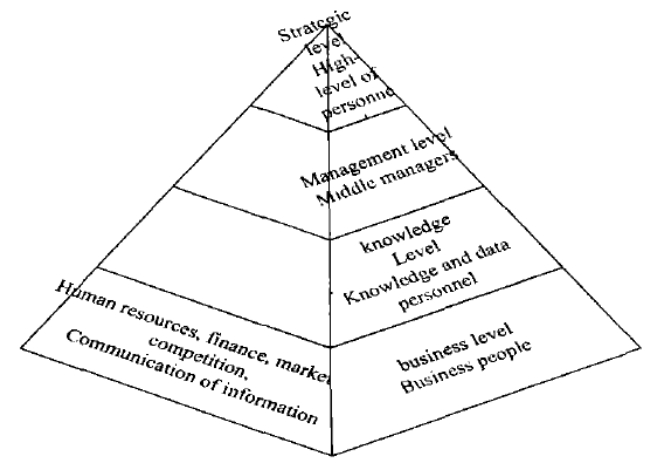

Fig. 1 Information systems in the different organizational levels

\section{The Design Process of Organization and Management Information System}

For the organization and management, based on the functions of the organization's management information system structure are the organization's functions and activities of the Organization hierarchy matrix description. The organization is the division of functions of the organization through the organization of the structural design. Design the organizational structure is not the only division of the functions of the organization is not the only one. For each functional department within an organization, have their own information needs, so need it to design an information system. This 
means that the organization functions in accordance with the need to use the information to establish management information systems, management information system to divide the functions of the organization subsystem.

System architecture design, distributed computing model (client-server computing), a variety of organizations build a business on the basis of communication networks, operating system services and database services.

The main functional structure of the Large-scale Sport Events organization and management information system is shown in Fig. 2.

In order to use the system more effective, We summarized the various functions of the organization of Large-scale Sport Events management system, grouped into 7 systems, These systems are Budget and Financial Management System, Risk Management System, Human Resource Management System, Logistics Management System, Communication of Information Management System, Marketing Management System, Competition Management System. The following discusses the main functions of these 7 systems.

The deployment of Large-scale Sport Events organization and management information systems is very easy. We only need to connect the server to the Internet, meanwhile the branches connect to the Internet through ADSL, cable broadband or dial-up Modem, and then this platform can be used. Therefore, this platform is easy to manage and maintain, and requires a low cost. The development of this platform generally adopts the user authentication methods. The overall design of the system adopts the modular development method. The system adopts B/S structure. The development of web pages is carried on by using ASP language, web page integration software using $\mathrm{C}++$ language and website background database using SQL Server 2005.

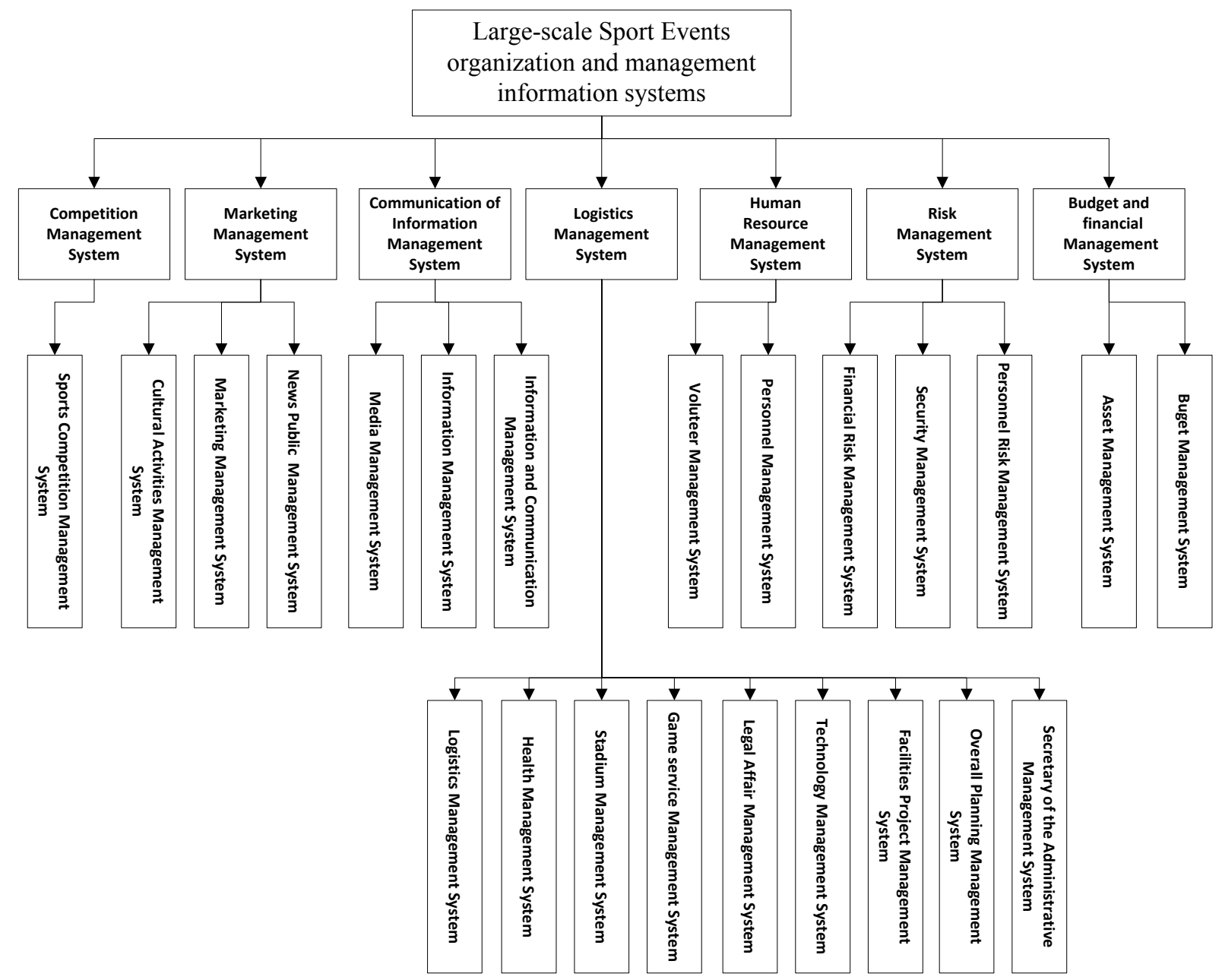

Fig. 2 The main functional structure of the organization and management information system

The design of the system is realized by JPS technology, architecture development mode of the combination of $\mathrm{B} / \mathrm{S}$ and $\mathrm{C} / \mathrm{S}, \mathrm{JDBC}$ connecting to data base and Struts technology. Struts is a frame 
technology base on MVC mode. The Action Servlet of the struts controls the navigation flow. Another concept, CURD operation, is introduced into Action; most of the operations are the following four kinds as creation, restoration, read and deletion in all the system. It is an application frame of WEB level, which has much higher configure ability and ever-increasing characteristic list. $\mathrm{B} / \mathrm{S}$ architecture, based on the multilevel application of J2EE, is mainly divided into presentation layer, service logic layer, system service layer and data storage layer. $\mathrm{C} / \mathrm{S}$ architecture divides the system of universities into two staple parts: the client and the server [5-6].

The communication between the client and server is taken in charge by RMI.

The data base of the university music education and teaching management system is based on SQL Server data base. The advantage of using SQL Server is that the SQL Server data base has remarkable simplification and availability and provides much powerful condition for smooth extension of the procedure [7].

The client is primarily responsible for human-computer interaction, including the relationship between the data and the application of graphics and the interface operations. Web server is mainly responsible for centralized management of client applications. The application server is primarily responsible for logical structure and data relationships of application systems, namely transaction processing. Depending on the application server, the application server can deal with their small business which is divided into multiple modules. The database server is primarily responsible for storing and organizing data, distributed management and synchronization of the various parts and so on [8].

\section{Conclusions}

The process of making the flow chart and PERT diagram of the events is the process of designing and planning the whole of organizing work. By use of flow chart large-scale events can be planned integrally, coordinated orderly and obtain the objectives set before hand with less time and resources. The study and making of flow chart provide a useful reference to planning and management of large-scale sports events and social activities. Large-scale Sport Events organization and management information systems is an important auxiliary tool for the work of Large-scale Sport Events organization, by using this system can effectively manage, control and coordination of the organization and management of Large-scale Sport Events.

\section{References}

[1] Won Kim. Cloud Computing: Status and Prognosis' Object Technology, vol. 8, no. 1, 2009, 2: $65-72$.

[2] Wang Chunxiang, Zhu Jun, Meng Lingkui. Modeling of the Interactive Virtual Classroom Based on VRML. Geospatial Information, 2009.

[3] Dianne Rahm. The role of information technology in building public administration theory, 1999.

[4] Federal Highway Administration, Managing Travel for Planned Special Events, Washington D.C., 2003.

[5] NCHRP. Transportation Planning and management for Special Events, A synthesis of highway practice. Transportation research Board. Washington D.C., 2003:32-41.

[6] ORTA. Nothing better than this (Transport for the Sydney 2000 Olympic and Paralympics Games). Sydney, Australia, OTRA, 2001.

[7] US Olympic Festival 1989 After Action Report: Transportation Planning, Coordination and Operations, Oklahoma City, Ok. 1989.

[8] Steven H. Abrams, Moving Crowds in Chicago: Baseball and The 4th Of July. Transportation Research Board. Washington, D.C. 2000. 